\title{
Asymmetric amination of cyclic $\beta$-keto esters catalyzed by amine- thiourea bearing multiple hydrogen bonding donors
}

\author{
Zhi-Hai Zhang, ${ }^{\text {a }}$ Xiu-Qin Dong, ${ }^{\text {a Hai-Yan Tao, }},{ }^{\text {a }}$ and Chun-Jiang Wanga,b* \\ ${ }^{a}$ College of Chemistry and Molecular Sciences, Wuhan University, Wuhan 430072, P. R. China \\ ${ }^{b}$ State Key Laboratory of Elemento-organic Chemistry, Nankai University, Tianjin, 300071, P. R. \\ China \\ E-mail: cjwang@whu.edu.cn
}

\begin{abstract}
Highly efficient asymmetric amination of cyclic $\beta$-keto esters with dialkyl azodicarboxylates has been achieved by bifunctional amine-thiourea bearing multiple hydrogen bond donors. Catalyst 1d showed excellent results for this transformation and provide optically active $\alpha$-amino acid derivatives in up to $96 \%$ ee. Multiple hydrogen bond donors play a significant role in accelerating reactions and improving enantioselectivities.
\end{abstract}

Keywords: Amination, asymmetric catalysis, organocatalyst, enantioselectivity, $\beta$-keto esters

\section{Introduction}

Optically active $\alpha$-amino acid derivatives are prevalent in many natural alkaloids, compounds of pharmaceutical significance, and biologically important building blocks in organic synthesis. ${ }^{1}$ The asymmetric amination of $\beta$-keto esters with azodicarboxylates provides an efficient approach for the construction of $\alpha, \alpha$-disubstituted amino acid derivatives containing a nitrogensubstituted quaternary stereocenter, ${ }^{2}$ and much attention has been paid to developing enantioselective catalytic protocols for this reaction over the past decade. Highly efficient $\mathrm{Cu}^{\mathrm{II}} / \mathrm{Ph}-\mathrm{BOX}$ and $\mathrm{Cu}^{\mathrm{II}} /$ trisoxazoline complexes catalyzed amination reaction of $\beta$-keto esters has

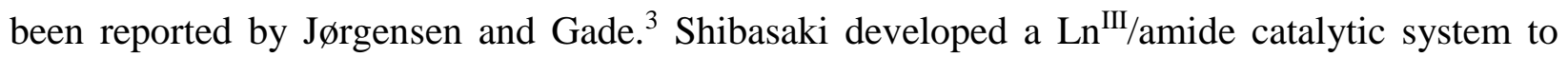
carry out this reaction with high reactivity and excellent enantioselectivity. ${ }^{4}$ Recently, asymmetric organocatalysis becomes a powerful and environmentally friendly methodology for the catalytic production of the valuable synthetic building blocks and has received much attention. $^{5}$ Cinchona alkaloid derivatives perform good to excellent reactivities and enantioselectivities for asymmetric amination of $\beta$-keto esters, $\beta$-keto lactones ${ }^{6}$ and $\alpha$ cyanoesters. $^{7}$ Takemoto's bifunctional thiourea catalyst exhibited excellent catalytic activity for this amination reaction. ${ }^{8}$ Axially chiral guanidines and quaternary phosphonium phase-transfer 
catalysts have also been designed and applied successfully for this transformation by Terada and Maruoka. ${ }^{9}$<smiles>C=CC1CN2CCC1C2[C@H](O)c1ccnc2ccccc12</smiles><smiles>C=CC1CC2CCN1C2[C@H](O)c1ccnc2ccccc12</smiles><smiles>CN(C)[C@H]1CCCC[C@H]1NC(=S)Nc1cc(C(F)(F)F)cc(C(F)(F)F)c1</smiles><smiles>CC(C)(C)Cc1cc(-c2ccc(C#[Al])cc2)cc(C(C)(C)C)c1</smiles><smiles>FC(F)(F)c1ccc(P(Br)(Br)(Br)Cc2c(-c3c(C(F)(F)F)c(-c4cc(C(F)(F)F)cc(C(F)(F)F)c4)cc4ccccc34)c(CP(Br)(Br)(Br)c3cc(C(F)(F)F)cc(C(F)(F)F)c3)cc3ccccc23)cc1</smiles>

Figure 1. Examples of some organocatalysts for asymmetric amination of $\beta$-keto ester.

Recently, we reported a new class of bifunctional amine-thiourea catalysts 1 bearing multiple hydrogen bond donors, which showed excellent performances in catalytic asymmetric Michael addition and nitro-Mannich reaction. ${ }^{10}$ Extending the interest of these organocatalysts in asymmetric catalysis, herein we report that oganocatalyst 1d shows excellent results for asymmetric amination of cyclic $\beta$-keto esters with azodicarboxylates and provides optically active $\alpha, \alpha$-disubstituted amino acid derivatives in up to $97 \%$ ee. 
<smiles>Cc1ccc(S(=O)(=O)N[C@H](c2ccccc2)[C@H](NC(=S)N[C]2CCCC[C@H]2N(C)C)c2ccccc2)cc1</smiles>

$\left(1 R, 2 R, 1^{\prime} S, 2 ' S\right)-1 \mathbf{a}$<smiles>Cc1ccc(N[C@H](NC(=S)N[C@H]2CCCC[C@H]2N(C)C)C(c2ccccc2)c2ccccc2)cc1</smiles>

$\left(1 R, 2 R, 1^{\prime} R, 2^{\prime} R\right)-\mathbf{1 b}$

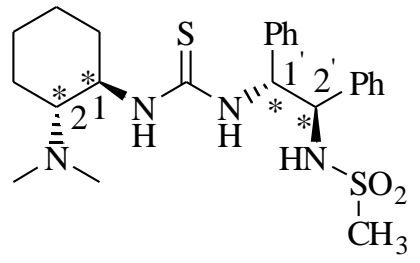

$\left(1 R, 2 R, 1^{\prime} R, 2^{\prime} R\right)-1 \mathbf{c}$

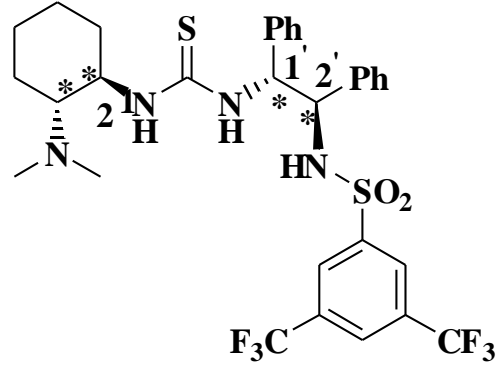

$\left(1 R, 2 R, 1^{\prime} R, 2^{\prime} R\right)-\mathbf{1 d}$

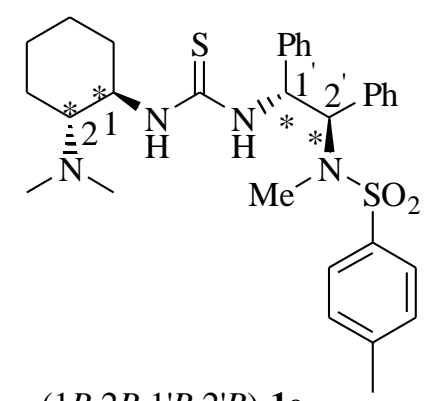

$\left(1 R, 2 R, 1^{\prime} R, 2^{\prime} R\right)-1 \mathbf{e}$

Figure 2. Amine-thioureas bearing multiple hydrogen bond donors.

\section{Results and Discussion}

Our initial investigation began with the amination of methyl 2-oxo-cyclopentanecarboxylate $2 \mathbf{a}$ and diethyl azodicarboxylate $\mathbf{3 a}$, and the representative results are summarized in Table 1.

To our delight, the reaction was finished in less than $10 \mathrm{~min}$ at room temperature in the presence of 10 mol\% fine-tunable multiple-hydrogen-bond-donor catalysts 1a-d (Table 1, entries 1-4). Among the catalysts tested, $\left(1 R, 2 R, 1^{\prime} R, 2^{\prime} R\right)$-1d bearing two electron-withdrawing $\mathrm{CF}_{3}$ groups on the aromatic ring of sulfonamide $\mathrm{NHSO}_{2} \mathrm{Ar}$ revealed to be the most efficient catalyst (Table 1, entry 4), which was consistent with the results achieved in the study of the Michael addition and the nitro-Mannich reaction. ${ }^{10}$ Much lower enantioselectivity was observed by the methylated $\left(1 R, 2 R, 1^{\prime} R, 2^{\prime} R\right)$-1e as the catalyst further demonstrated the significant importance of the multiple hydrogen bond donors embedded in the catalysts on the reactivity and enantioselectivity (Table 1, entry 6). The catalyst loading is crucial for the reproducibility of the experiment. The enantioselectivity decreased from $81 \%$ to $54 \%$ when the catalyst loading was reduced from $10 \mathrm{~mol} \%$ to $5 \mathrm{~mol} \%$ (Table 1 , entrie 4 and 5). ${ }^{11}$ A preliminary screening of solvent

effects showed that EtOAc, THF and ether were the solvents of choice; other solvents such as DCM, PhMe, $\mathrm{CH}_{3} \mathrm{CN}$, DMSO gave lower enantioselectivities (Table 1, entries 4 and 7-12). Protic solvents such as ${ }^{i} \mathrm{PrOH}$ or $\mathrm{EtOH}$ produced almost racemic adducts (Table 1, entries 13 and 14), which could be ascribed to the unfavorable background reaction ${ }^{6}$ or the competitive 
activation of $3 \mathrm{a}$ between catalyst $\mathbf{1 d}$ and the protic solvent. Reducing the temperature to $-78{ }^{\circ} \mathrm{C}$ led to a complete reaction with a remarkable improvement of enantioselectivity (Table 1, entries 15 and 16).

Table 1. Screening studies of asymmetric amination of methyl 2-oxocyclopentanecarboxylate 2a with diethyl azodicarboxylate $\mathbf{3 a}$ catalyzed by multiple-hydrogen-bond-donor organocatalysts $\mathbf{1}^{\mathrm{a}}$

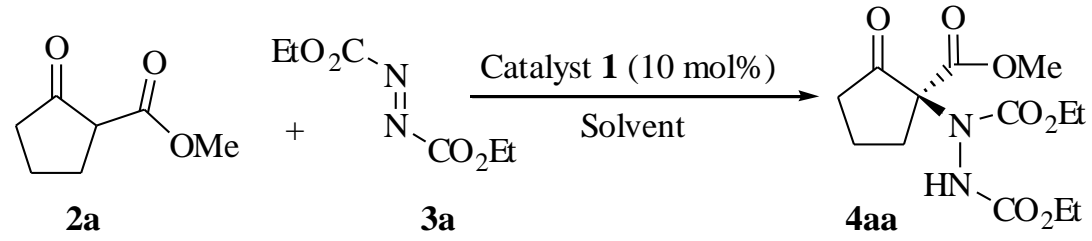

\begin{tabular}{|c|c|c|c|c|c|c|}
\hline Entry & Catal. & solvent & $\mathrm{T}\left({ }^{\circ} \mathrm{C}\right)$ & time (min) & yield $(\%)^{b}$ & ee $(\%)^{\mathrm{c}, \mathrm{d}}$ \\
\hline 1 & $1 \mathbf{a}$ & EtOAc & $\mathrm{rt}$ & 10 & 94 & 68 \\
\hline 2 & $1 b$ & EtOAc & $\mathrm{rt}$ & 10 & 96 & 73 \\
\hline 3 & 1c & EtOAc & $\mathrm{rt}$ & 10 & 96 & 76 \\
\hline 4 & 1d & EtOAc & $\mathrm{rt}$ & 10 & 95 & 81 \\
\hline 5 & 1e & EtOAc & $\mathrm{rt}$ & 30 & 91 & 54 \\
\hline 6 & $1 d$ & EtOAc & $\mathrm{rt}$ & 60 & 96 & 43 \\
\hline 7 & 1d & $\mathrm{THF}$ & $\mathrm{rt}$ & 10 & 93 & 79 \\
\hline 8 & 1d & $\mathrm{Et}_{2} \mathrm{O}$ & $\mathrm{rt}$ & 10 & 95 & 80 \\
\hline 9 & 1d & $\mathrm{PhMe}$ & $\mathrm{rt}$ & 10 & 95 & 67 \\
\hline 10 & 1d & DCM & $\mathrm{rt}$ & 30 & 92 & 41 \\
\hline 11 & 1d & $\mathrm{MeCN}$ & $\mathrm{rt}$ & 20 & 88 & 42 \\
\hline 12 & 1d & DMSO & $\mathrm{rt}$ & 10 & 91 & 20 \\
\hline 13 & 1d & $\mathrm{PrOH}$ & $\mathrm{rt}$ & 10 & 86 & 0 \\
\hline 14 & $1 d$ & $\mathrm{EtOH}$ & $\mathrm{rt}$ & 10 & 92 & 0 \\
\hline 15 & 1d & $\mathrm{Et}_{2} \mathrm{O}$ & -78 & 60 & 94 & 92 \\
\hline 16 & 1d & EtOAc & -78 & 60 & 95 & 95 \\
\hline
\end{tabular}

${ }^{a}$ Unless otherwise noted, the reaction was performed with $0.10 \mathrm{mmol}$ of $1 \mathrm{a}$ and $0.125 \mathrm{mmol}$ of 2a in $0.5 \mathrm{~mL}$ of solvent. ${ }^{\mathrm{b}}$ Isolated yield. ${ }^{\mathrm{c}}$ Enantiomeric excesses were determined by chiral HPLC analysis. ${ }^{\mathrm{d}}$ The absolute configurations of the product was determined as $\mathrm{S}$ by comparing the optical rotation and HPLC retention time with the reported date. ${ }^{7 e} 5 \mathrm{~mol} \%$ catalyst was used. 
Table 2. Enantioselective amination of various $\beta$-ketoesters $\mathbf{2}$ with dialkyl azodicarboxylates $\mathbf{3}$ catalyzed by organocatalyst $\mathbf{1 d}^{\mathrm{a}}$<smiles>[R20]OC(=O)N=NNC(=O)O[R]</smiles>

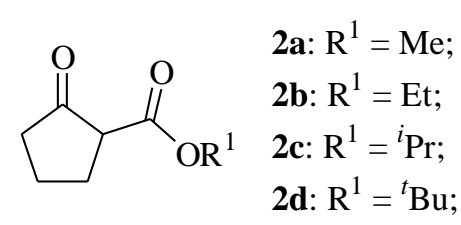<smiles>CCOC(=O)C1CCCCC1=O</smiles>

$2 \mathrm{e}$<smiles>CCOC(=O)C1CCCCCC1=O</smiles><smiles>CCOC(=O)C1Cc2ccccc2C1=O</smiles>

$2 \mathrm{~g}$<smiles>CCOC(=O)C1CCc2ccccc2C1=O</smiles>

$2 h$<smiles>[R]OC(=O)N=NC([R])=O</smiles>

3a: $\mathrm{R}^{2}=\mathrm{Et}$;

3b: $\mathrm{R}^{2}={ }^{i} \mathrm{Pr}$;

3c: $\mathrm{R}^{2}={ }^{t} \mathrm{Bu}$;

\begin{tabular}{ccccccc}
\hline Entry & $\mathbf{2}$ & $\mathbf{3}$ & Prod. $\mathbf{4}$ & time $(\mathrm{h})$ & yield $(\%)^{\mathrm{b}}$ & ee $(\%)^{\mathrm{c}}$ \\
\hline 1 & $\mathbf{2 a}$ & $\mathbf{3 a}$ & $\mathbf{4 a b}$ & 1 & 98 & 95 \\
2 & $\mathbf{2 a}$ & $\mathbf{3 b}$ & $\mathbf{4 a b}$ & 1 & 96 & 96 \\
3 & $\mathbf{2 a}$ & $\mathbf{3 c}$ & $\mathbf{4 a c}$ & 1 & 96 & 95 \\
4 & $\mathbf{2 b}$ & $\mathbf{3 b}$ & $\mathbf{4 b b}$ & 1 & 97 & 97 \\
5 & $\mathbf{2 c}$ & $\mathbf{3 b}$ & $\mathbf{4 c b}$ & 3 & 95 & 93 \\
6 & $\mathbf{2 d}$ & $\mathbf{3 b}$ & $\mathbf{4 d b}$ & 3 & 98 & 94 \\
7 & $\mathbf{2 b}$ & $\mathbf{3 c}$ & $\mathbf{4 b c}$ & 3 & 97 & 94 \\
8 & $\mathbf{2 c}$ & $\mathbf{3 c}$ & $\mathbf{4 c c}$ & 6 & 95 & 90 \\
9 & $\mathbf{2 e}$ & $\mathbf{3 a}$ & $\mathbf{4 c a}$ & 10 & 88 & 73 \\
10 & $\mathbf{2 f}$ & $\mathbf{3 a}$ & $\mathbf{4 f a}$ & 1.5 & 91 & 74 \\
11 & $\mathbf{2 g}$ & $\mathbf{3 c}$ & $\mathbf{4 g e}$ & 3 & 86 & 76 \\
12 & $\mathbf{2 h}$ & $\mathbf{3 a}$ & $\mathbf{4 h a}$ & 3 & 94 & 69 \\
\hline
\end{tabular}

a Unless otherwise noted, the reaction was performed with $0.10 \mathrm{mmol}$ of $2,0.125 \mathrm{mmol}$ of $\mathbf{3}$ in $0.5 \mathrm{~mL}$ of EtOAc. ${ }^{\mathrm{b}}$ Isolated yield. ${ }^{\mathrm{c}}$ Enantiomeric excesses were determined by chiral HPLC analysis. ${ }^{\mathrm{d}}$ The absolute configurations of the product was determined as $S$ by comparing the optical rotation and HPLC retention time with the reported date. ${ }^{7}$ 
Having established the optimal reaction conditions, the scope of this amination reaction was investigated, and the results are summarized in Table 2. First, we examined the effect of the ester substituent of the azodicarboxylates. The results show that the steric hindrance of the ester groups has no influence on the enantioselectivities, and excellent ee ranging from 95\% to 96\% were observed for all the tested azodicarboxylates, which was opposite to the trend exhibited by Takemoto's thiourea catalyst (Table 2, entries 1-3). Subsequently, the effect of the ester functional group of 2-oxo-cyclopentanecarboxylate $\mathbf{2}$ was also investigated for this transformation. Although no obvious differentiation of enantioselectivity was observed when the ester functional group of $\mathbf{2}$ switched from methyl to ethyl, iso-propyl or tert-butyl, the bulky ester functional group such as iso-propyl and tert-butyl has a detrimental effect on the reactivity and renders an extended reaction time (Table 2, entries 2 and 4-6). Catalyst 1d could also affect the asymmetric amination of both six- an seven-membered ring substrates $\mathbf{2 e}$ and $\mathbf{2} \mathbf{f}$, although moderate enantioselectivities were achieved (Table 2, entries 9 and 10). Bicyclic $\beta$-keto esters $\mathbf{2 g}$ and $\mathbf{2 h}$ were also tolerated in this reaction, and the corresponding adducts could be obtained in high yields with $76 \%$ and $69 \%$ ee, respectively (Table 2, entries 11 and 12). The results for asymmetric amination of cyclic $\beta$-keto esters are comparable to those obtained with chiral metal catalysts or organocatalysts. ${ }^{3-9}$<smiles>CC(=O)C1CCCC1=O</smiles>

$2 \mathrm{i}$

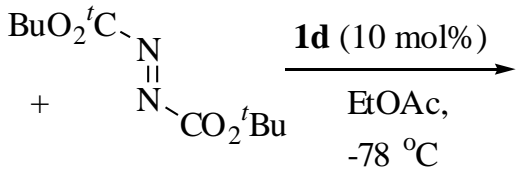

3c

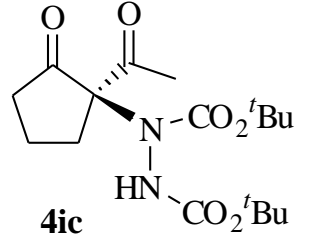

$95 \%$ yield

$80 \%$ ee

$2 \mathrm{j}$<smiles>CC(=O)C(C)C(=O)O</smiles><smiles>O=C(O)COC(=O)N=NC(=O)OCc1ccccc1</smiles>

$3 c$<smiles>CCOC(=O)C(C)(C(C)=O)N(NC(=O)OCC(C)(C)C)C(=O)OCC(C)(C)C</smiles>

$79 \%$ yield

$10 \%$ ee

Scheme 1. Asymmetric amination of 2-acetyl-cyclopentanone 2i and ethyl 2-methyl-3-oxobutylate $\mathbf{2 j}$ with dialkyl azodicarboxylate.

The asymmetric amination of 2-acetylcyclopentanone was also investigated. As shown in scheme 1, the desired adduct 4ia was generated in $95 \%$ yield with $80 \%$ ee under the optimized reaction condition. However, only $10 \%$ ee was achieved when acyclic $\beta$-keto ester $\mathbf{2 j}$ c was applied in this system. 


\section{Conclusions}

In summary, we have described a highly efficient asymmetric amination of cyclic $\beta$-keto esters with dialkyl azodicarboxylates catalyzed by bifunctional amine-thiourea catalyst bearing multiple hydrogen bond donors. Catalyst 1d exhibits the best performance for this transformation and provides optically active $\alpha$-amino acid derivatives in up to $96 \%$ ee. Multiple hydrogen bond donors play a significant role in accelerating reactions and improving enantioselectivities. Future investigation of those bifunctional organocatalyst in other asymmetric reactions is ongoing in our laboratory and will be reported in due course.

\section{Experimental Section}

General. ${ }^{1} \mathrm{H}$ NMR spectra were recorded on a VARIAN Mercury $300 \mathrm{MHz}$ spectrometer in chloroform- $\mathrm{d}_{3}$. Chemical shifts are reported in ppm with the internal TMS signal at $0.0 \mathrm{ppm}$ as a standard. The data are reported as $(\mathrm{s}=$ singlet, $\mathrm{d}=$ doublet, $\mathrm{t}=$ triplet, $\mathrm{q}=$ quartet, $\mathrm{m}=$ multiplet or unresolved, brs = broad singlet, coupling constant(s) in $\mathrm{Hz}$, integration). ${ }^{13} \mathrm{C}$ NMR spectra were recorded on a VARIAN Mercury $75 \mathrm{MHz}$ spectrometer in chloroform- $d_{3}$. Chemical shifts are reported in ppm with the internal chloroform signal at $77.0 \mathrm{ppm}$ as a standard. Commercially obtained reagents were used without further purification. All reactions were monitored by TLC with silica gel-coated plates. Enantiomeric ratios were determined by HPLC, using a chiralpak AS-H column, a chiralpak AD-H column or a chiralcel OD-H column with hexane and $i$-PrOH as solvents.

\section{Synthesis of $\quad N$-((1R,2R)-2-amino-1,2-diphenylethyl)-3,5-bis(trifluoromethyl)-benzene- sulfonamide}<smiles>NC(N)c1ccccc1</smiles><smiles>O=S(=O)(Cl)c1cc(C(F)(F)F)cc(C(F)(F)F)c1</smiles>
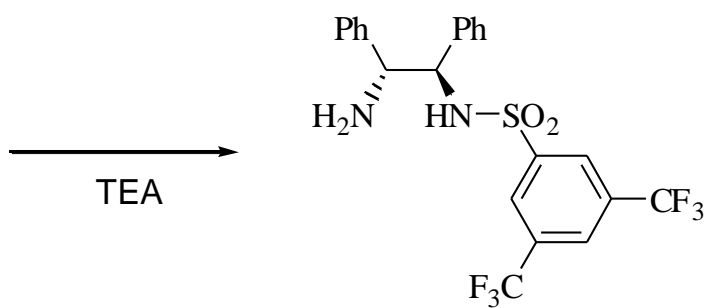

A solution of 3,5-bis(trifluoromethyl) benzenesulfonyl chloride (312.6 $\mathrm{mg}, 1 \mathrm{mmol}$ ) in anhydrous THF $(5 \mathrm{~mL})$ was added dropwise to $(1 R, 2 R)$-1,2-diphenylethane-1,2- diamine (212.3mg, 1 mmol), triethylamine $(277 \mathrm{~mL}, 2 \mathrm{mmol})$ and anhydrous THF $(10 \mathrm{~mL})$ with icecooling. The reaction mixture was brought to room temperature and stirred over night. The result solution was diluted with ethyl acetate and washed with brine. The resulted organic phase was dried over $\mathrm{Na}_{2} \mathrm{SO}_{4}$ and concentrated in vacuo. The residue was purified by flash silica gel chromatography. The pure product was obtained as a white solid in $86 \%$ yield. Mp. $158-160{ }^{\circ} \mathrm{C}$; $[\mathrm{R}]^{25} \mathrm{D}+15.6\left(c, 0.4, \mathrm{CHCl}_{3}\right)$; IR (KBr) 3436, 3355, 3298, 1352, 1278, 1265, 1196, 1163, 1128 
$\mathrm{cm}^{-1} ;{ }^{1} \mathrm{H} \mathrm{NMR}\left(\mathrm{CDCl}_{3}, \mathrm{TMS}, 300 \mathrm{MHz}\right) \delta 4.18(\mathrm{~d}, J=4.5 \mathrm{~Hz}, 1 \mathrm{H}), 4.52(\mathrm{~d}, J=4.5 \mathrm{~Hz}, 1 \mathrm{H})$, $7.13(\mathrm{~s}, 10 \mathrm{H}), 7.79(\mathrm{~s}, 1 \mathrm{H}), 7.86(\mathrm{~s}, 2 \mathrm{H}) ;{ }^{13} \mathrm{C} \mathrm{NMR}\left(\mathrm{CDCl}_{3}, \mathrm{TMS}, 75 \mathrm{MHz}\right) \delta$ 60.27, 63.68, 125.69, 126.42, 127.17, 127.32, 128.10, 128.18, 128.76, 132.17, 132.62, 138.27, 141.15, 143.40; HRMS Calcd. for $\mathrm{C}_{22} \mathrm{H}_{19} \mathrm{~F}_{6} \mathrm{~N}_{2} \mathrm{O}_{2} \mathrm{~S}: 489.1071$, found: 489.1047;

\section{Synthesis of $(1 R, 2 R)$-2-isothiocyanato- $N, N$-dimethylcyclohexanamine}

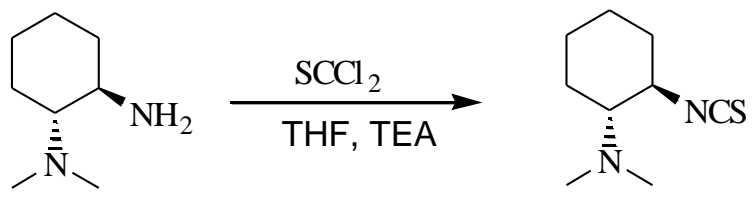

Thiophosgene $(1.86 \mathrm{~mL}, 24.4 \mathrm{mmol})$ was added dropwise to a solution of $(1 R, 2 R)$ - $N, N$-dimethylcyclohexane-1,2-diamine $(2.31 \mathrm{~g}, 16.3 \mathrm{mmol})$ and triethylamine $(6.77 \mathrm{~mL}, 48.8 \mathrm{mmol})$ with icecooling. The reaction mixture was stirred for about $4 \mathrm{~h}$, and TLC analysis indicated completion of the reaction. Then the reaction mixture was concentrated in vacuo, and the residue was purified by flash silica gel chromatography to afford brown oil in $91 \%$ yield. $[\alpha]^{25} \mathrm{D}-99.0\left(c 0.36, \mathrm{CHCl}_{3}\right)$; IR (KBr) 2935, 2860, 2827, 2780, 2185, 2095, $1618 \mathrm{~cm}^{-1} ;{ }^{1} \mathrm{H} \mathrm{NMR}\left(\mathrm{CDCl}_{3}, \mathrm{TMS}, 300 \mathrm{MHz}\right) \delta$ 1.16-1.25 (m, 4H), 1.69-1.84 (m, 3H), 2.15-2.19 (m, 1H), $2.34(\mathrm{~s}, 6 \mathrm{H}), 2.40-2.47(\mathrm{~m}, 1 \mathrm{H}), 3.51-$ $3.59(\mathrm{~m}, 1 \mathrm{H}) ;{ }^{13} \mathrm{C} \mathrm{NMR}\left(\mathrm{CDCl}_{3}\right.$, TMS, $\left.75 \mathrm{MHz}\right) \delta 23.58,24.32,24.45,33.57,40.41,58.23$, 67.22, 76.63, 77.06, 77.48; $\mathrm{MS}(\mathrm{EI}) \mathrm{m} / z 184\left([\mathrm{M}]^{+*}\right)$.

\section{General procedure for the synthesis of organocatalysts (1a-1d)}

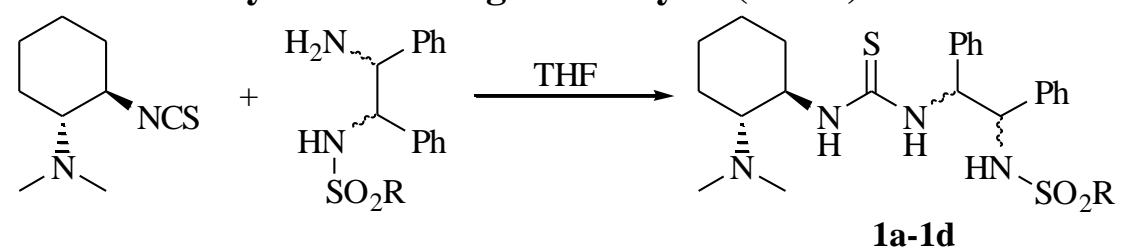

To a solution of corresponding sulfonamide $(1 \mathrm{mmol})$ in anhydrous THF $(10 \mathrm{~mL})$ was added $(1 R, 2 R)$-2-isothiocyanato- $N, N$-dimethylcyclohexanamine $(194 \mathrm{mg}, 1.05 \mathrm{mmol})$ at room temperature. The solution was stirred overnight. TLC analysis indicated completion of the reaction. The reaction mixture was concentrated in vacuo. The residue was purified by flash silica gel chromatography.

1-((1R,2R)-2-(Dimethylamino)cyclohexyl)-3-((1S,2S)-1,2-diphenyl-2-(tosylamino)ethyl) thiourea (1a)<smiles>Cc1ccc(NS(=O)(=O)c2ccccc2)cc1</smiles> 
The pure product was obtained as a white solid in $95 \%$ yield. Mp. $110-113{ }^{\circ} \mathrm{C} ;[\alpha]^{25} \mathrm{D}+18.5(c$ 0.62, $\mathrm{CHCl}_{3}$ ); IR (KBr) 3357, 3062, 3030, 2932, 2858, 1536, 1327, $1155 \mathrm{~cm}^{-1} ;{ }^{1} \mathrm{H} \mathrm{NMR}\left(\mathrm{CDCl}_{3}\right.$, TMS, $300 \mathrm{MHz}) \delta 1.08-1.29(\mathrm{~m}, 4 \mathrm{H}), 1.63-1.76(\mathrm{~m}, 3 \mathrm{H}), 1.95(\mathrm{~s}, 6 \mathrm{H}), 2.20(\mathrm{~m}, 1 \mathrm{H}), 2.25(\mathrm{~s}$, $3 \mathrm{H}), 2.42(\mathrm{~m}, 1 \mathrm{H}), 3.58(\mathrm{~m}, 1 \mathrm{H}), 4.70(\mathrm{~d}, J=10.2 \mathrm{~Hz}, 1 \mathrm{H}), 5.68(\mathrm{~m}, 1 \mathrm{H}), 6.86-7.13(\mathrm{~m}, 12 \mathrm{H})$, $7.41(\mathrm{~d}, J=8.1 \mathrm{~Hz}, 2 \mathrm{H}) ;{ }^{13} \mathrm{C} \mathrm{NMR}\left(\mathrm{CDCl}_{3}, \mathrm{TMS}, 75 \mathrm{MHz}\right) \delta 21.59,22.37,24.79,25.17,33.41$, 40.13, 56.67, 62.99, 63.84, 67.25, 127.10, 127.40, 128.03, 128.13, 128.68, 129.18, 138.21, 138.50, 142.54, 183.00; HRMS Calcd. for $\mathrm{C}_{30} \mathrm{H}_{39} \mathrm{~N}_{4} \mathrm{O}_{2} \mathrm{~S}_{2}: 551.2514$, found: 551.2494.

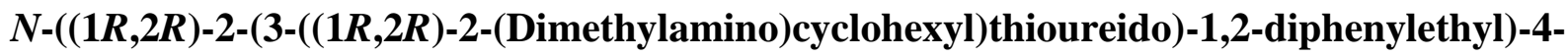
methylbenzenesulfonamide (1b)

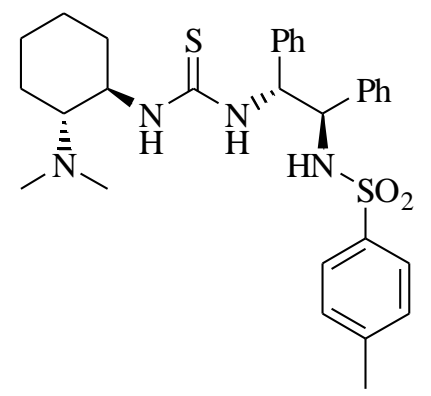

The pure product was obtained as a white solid in $80 \%$ yield. Mp. $128-130{ }^{\circ} \mathrm{C} ;[\alpha]^{25} \mathrm{D}+3.5(c$ $\left.0.62, \mathrm{CHCl}_{3}\right)$; IR (KBr) 3355, 3061, 3030, 2932, 2859, 1538, 1454, 1329, $1157 \mathrm{~cm}^{-1}$; ${ }^{1} \mathrm{H}$ NMR $\left(\mathrm{CDCl}_{3}, \mathrm{TMS}, 300 \mathrm{MHz}\right) \delta 1.18-1.27(\mathrm{~m}, 4 \mathrm{H}), 1.68-1.90(\mathrm{~m}, 3 \mathrm{H}), 2.30(\mathrm{~s}, 1 \mathrm{H}), 2.35(\mathrm{~s}, 6 \mathrm{H})$, 2.20-2.43 (m, 2H), $3.47(\mathrm{~s}, 1 \mathrm{H}), 4.72(\mathrm{~d}, J=10.8 \mathrm{~Hz}, 1 \mathrm{H}), 5.81(\mathrm{~m}, 1 \mathrm{H}), 6.86-7.21(\mathrm{~m}, 12 \mathrm{H})$, $7.40(\mathrm{~d}, J=10.8 \mathrm{~Hz}, 2 \mathrm{H}) ;{ }^{13} \mathrm{C} \mathrm{NMR}\left(\mathrm{CDCl}_{3}, \mathrm{TMS}, 75 \mathrm{MHz}\right) \delta 21.67,22.88,24.76,25.11,32.89$, 41.29, 56.85, 63.04, 64.79, 67.17, 126.99, 127.37, 127.95, 128.10, 128.67, 129.28, 138.46, 142.62, 182.70; HRMS Calcd. for $\mathrm{C}_{30} \mathrm{H}_{39} \mathrm{~N}_{4} \mathrm{O}_{2} \mathrm{~S}_{2}$ : 551.2514, found: 551.2499.

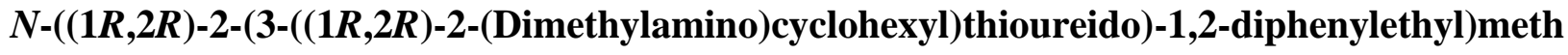
anesulfonamide (1c)<smiles>COS(=O)(=O)NC(c1ccccc1)[C@H](NC(=S)N[C@H]1CCCC[C@H]1N(C)C)c1ccccc1</smiles>

The pure product was obtained as a white solid in $97 \%$ yield. Mp. $108-110{ }^{\circ} \mathrm{C} ;[\alpha]^{25} \mathrm{D}+9.4(c$ 0.44, $\mathrm{CHCl}_{3}$ ); IR (KBr) 3356, 3062, 2931, 2858, 1545, 1320, $1148 \mathrm{~cm}^{-1}$; ${ }^{1} \mathrm{H} \mathrm{NMR}\left(\mathrm{CDCl}_{3}, \mathrm{TMS}\right.$, $300 \mathrm{MHz}) \delta 1.21-1.24(\mathrm{~m}, 4 \mathrm{H}), 1.69-1.90(\mathrm{~m}, 4 \mathrm{H}), 2.20(\mathrm{~m}, 1 \mathrm{H}), 2.41(\mathrm{~s}, 6 \mathrm{H}), 2.47(\mathrm{~s}, 3 \mathrm{H}), 3.70$ $(\mathrm{m}, 1 \mathrm{H}), 4.79(\mathrm{~d}, J=10.2 \mathrm{~Hz}, 1 \mathrm{H}), 5.88(\mathrm{~s}, 1 \mathrm{H}), 7.08-7.22(\mathrm{~m}, 10 \mathrm{H}) ;{ }^{13} \mathrm{C} \mathrm{NMR}\left(\mathrm{CDCl}_{3}, \mathrm{TMS}, 75\right.$ $\mathrm{MHz}) \delta 22.65,24.37,24.52,32.59$, 39.86, 41.69, 55.24, 62.25, 64.38, 66.34, 127.64, 127.76, 128.44, 128.54, 138.22, 139.15, 183.50; HRMS Calcd. for $\mathrm{C}_{24} \mathrm{H}_{35} \mathrm{~N}_{4} \mathrm{O}_{2} \mathrm{~S}_{2}: 475.2201$, found: 475.2168 . 
$N$-((1R,2R)-2-(3-((1R,2R)-2-(Dimethylamino)cyclohexyl)thioureido)-1,2-diphenylethyl)-3,5bis(trifluoromethyl)benzenesulfonamide (1d)<smiles>CN(C)[C@@H]1CCCC[C@H]1NC(=S)N[C@H](c1ccccc1)[C@H](NS(=O)(=O)c1ccccc1)c1ccccc1</smiles>

The pure product was obtained as a white solid in $99 \%$ yield. Mp. $168-170{ }^{\circ} \mathrm{C} ;[\alpha]^{25} \mathrm{D}+43.6(c$ 0.34, $\mathrm{CHCl}_{3}$ ); IR (KBr) 3423, 2936, 1541, 1539, 1279, 1162, $1141 \mathrm{~cm}^{-1}$; ${ }^{1} \mathrm{H} \mathrm{NMR}\left(\mathrm{CDCl}_{3}, \mathrm{TMS}\right.$, $300 \mathrm{MHz}) \delta 1.20(\mathrm{~m}, 4 \mathrm{H}), 1.71-1.94(\mathrm{~m}, 3 \mathrm{H}), 2.18(\mathrm{~m}, 1 \mathrm{H}), 2.42(\mathrm{~s}, 6 \mathrm{H}), 2.44(\mathrm{~m}, 1 \mathrm{H}), 3.35(\mathrm{~m}$, $1 \mathrm{H}), 4.81-4.86(\mathrm{~m}, 1 \mathrm{H}), 5.87(\mathrm{~m}, 1 \mathrm{H}), 6.86-7.12(\mathrm{~m}, 10 \mathrm{H}), 7.71(\mathrm{~s}, 1 \mathrm{H}), 7.83(\mathrm{~s}, 2 \mathrm{H}) ;{ }^{13} \mathrm{C}$ NMR $\left(\mathrm{CDCl}_{3}, \mathrm{TMS}, 75 \mathrm{MHz}\right) \delta 24.65,24.98,24.76,33.00,41.77,60.34,63.04,63.98,65.39,67.79$, $120.79,124.41,125.26,125.59,126.49,127.20,127.88,128.03,128.25,128.40,128.73,128.86$, 131.89, 132.35, 136.97, 137.76, 144.45, 183.27; HRMS Calcd. for $\mathrm{C}_{31} \mathrm{H}_{35} \mathrm{~F}_{6} \mathrm{~N}_{4} \mathrm{O}_{2} \mathrm{~S}_{2}$ : 673.2106, found: 673.2058 .

Synthesis of $\quad N-((1 R, 2 R)-2-(3-((1 R, 2 R)-2-($ dimethylamino $)$ cyclohexyl)thioureido)- $\quad 1,2-$ diphenylethyl)- $N, 4-$ dimethylbenzenesulfonamide (1e)

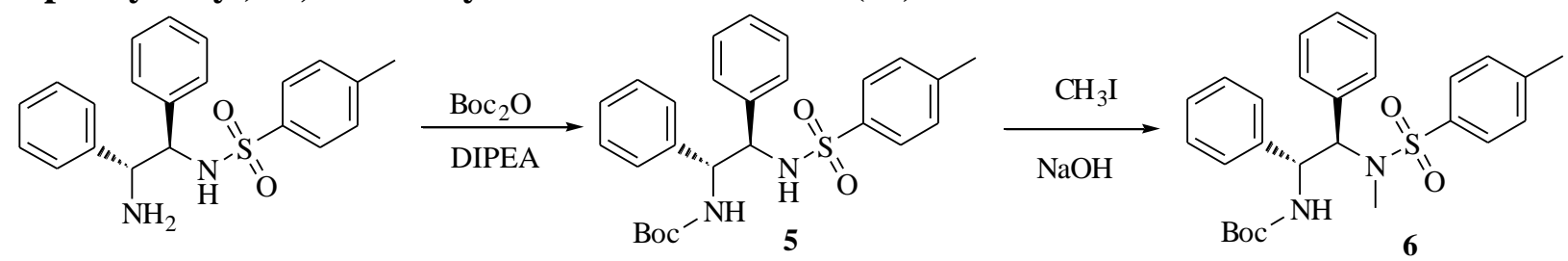<smiles>Cc1ccc(S(=O)(=O)N(C)[C@H](c2ccccc2)[C@H](N)c2ccccc2)cc1</smiles>

7

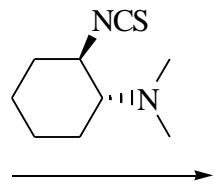<smiles>Cc1ccc(S(=O)(=O)N(C)[C@H](c2ccccc2)[C@H](NC(=S)N[C@H]2CCCC[C@H]2N(C)C)c2ccccc2)cc1</smiles> 
Synthesis of tert-butyl (1R,2R)-1,2-diphenyl-2-(tosylamino)ethylcarbamate (5)<smiles>Cc1ccc(S(=O)(=O)N[C@H](c2ccccc2)[C@H](NC(=O)OCc2ccccc2)c2ccccc2)cc1</smiles>

A solution of $\mathrm{Boc}_{2} \mathrm{O}(94.3 \mathrm{mg}, 0.432 \mathrm{~mol})$ in anhydrous dichloromethane $(10 \mathrm{~mL})$ was added to $(1 R, 2 R)$-1,2-diphenyl- $N$-tosylethane-1,2-diamine $(144 \mathrm{mg}, 0.393 \mathrm{mmol})$ and DIPEA $(74.3 \mu \mathrm{L}$, $0.432 \mathrm{mmol})$ in anhydrous dichloromethane $(20 \mathrm{~mL})$ with ice-cooling. After the addition, the reaction mixture was brought to room temperature and stirred overnight. TLC analysis indicated completion of the reaction. The reaction mixture was concentrated in vacuo. The residue was purified by flash silica gel chromatography. The pure product was obtained as a white solid in 90\% yield. Mp. 167-170 ${ }^{\circ} \mathrm{C}$; $[\alpha]^{25} \mathrm{D}+13.0$ (c 0.78, $\mathrm{CHCl}_{3}$ ); IR (KBr) 3390, 3312, 2928, 1686, $1514,1158 \mathrm{~cm}^{-1} ;{ }^{1} \mathrm{H} \mathrm{NMR}\left(\mathrm{CDCl}_{3}\right.$, TMS, $\left.300 \mathrm{MHz}\right) \delta 1.45(\mathrm{~s}, 9 \mathrm{H}), 2.30(\mathrm{~s}, 3 \mathrm{H}), 4.56-4.61(\mathrm{~m}$, $1 \mathrm{H}), 4.78-4.84(\mathrm{~m}, 1 \mathrm{H}), 5.33(\mathrm{br}, 1 \mathrm{H}), 6.22(\mathrm{br}, 1 \mathrm{H}), 6.78-6.81(\mathrm{~m}, 2 \mathrm{H}), 6.96-7.02(\mathrm{~m}, 7 \mathrm{H}), 7.14-$

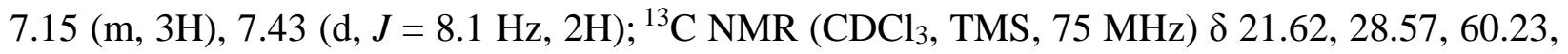
64.16, 80.79, 127.11, 127.54, 12769, 128.03, 128.22, 128.72, 129.33, 138.08, 138.57, 142.88, 156.55; HRMS Calcd. for $\mathrm{C}_{26} \mathrm{H}_{30} \mathrm{~N}_{2} \mathrm{O}_{4} \mathrm{~S}+\mathrm{Na}^{+}$: 489.1824 , found: 489.1827 .

\section{Synthesis of tert-butyl $(1 R, 2 R)-2-(N$-methyl- $N$-tosylamino)-1,2-diphenyl ethylcarbamate (6)}<smiles>Cc1ccc(S(=O)(=O)N(C)[C@H](c2ccccc2)[C@H](NC(=O)OCc2ccccc2)c2ccccc2)cc1</smiles>

Methyl iodide $(220 \mu \mathrm{L}, 3.54 \mathrm{mmol})$ was added to a solution of tert-butyl (1R,2R)-1,2-diphenyl2-(tosylamino)ethylcarbamate $(165 \mathrm{mg}, 0.35 \mathrm{mmol})$ and $1 \mathrm{~N} \mathrm{NaOH}(0.4 \mathrm{~mL})$ in 1,4-dioxane $(2$ $\mathrm{mL}$ ) at room temperature. TLC analysis indicated completion of the reaction after about $4 \mathrm{~h}$. The result solution was diluted with water and extracted with ethyl acetate. The resulted oil phase was washed with brine, dried with $\mathrm{Na}_{2} \mathrm{SO}_{4}$ and concentrated in vacuo. The residue was purified by flash silica gel chromatography. The pure product was obtained as a white solid in $81 \%$ yield. Mp. 67-69 ${ }^{\circ} \mathrm{C} ;[\alpha]^{25} \mathrm{D}-30.4\left(c 0.54, \mathrm{CHCl}_{3}\right)$; IR (KBr) 3428, 2975, 2928, 1711, 1599, 1508, 1384, $1163 \mathrm{~cm}^{-1} ;{ }^{1} \mathrm{H}$ NMR $\left(\mathrm{CDCl}_{3}, \mathrm{TMS}, 300 \mathrm{MHz}\right) \delta 1.43(\mathrm{~s}, 9 \mathrm{H}), 2.36(\mathrm{~s}, 3 \mathrm{H}), 2.80(\mathrm{~s}, 3 \mathrm{H}), 5.20(\mathrm{~m}$, $1 \mathrm{H}), 5.32(\mathrm{~m}, 1 \mathrm{H}), 5.45(\mathrm{br}, 1 \mathrm{H}), 6.94-7.20(\mathrm{~m}, 12 \mathrm{H}), 7.58(\mathrm{~d}, J=6.0 \mathrm{~Hz}, 2 \mathrm{H}) ;{ }^{13} \mathrm{C} \mathrm{NMR}$ $\left(\mathrm{CDCl}_{3}, \mathrm{TMS}, 75 \mathrm{MHz}\right) \delta 21.69,28.59,30.07,54.18,64.67,80.00,127.34,127.64,127.79$, 128.28, 128.37, 128.68, 129.33, 129.79, 134.73, 137.56, 140.20, 143.39, 155.81; HRMS Calcd. for $\mathrm{C}_{27} \mathrm{H}_{32} \mathrm{~N}_{2} \mathrm{O}_{4} \mathrm{~S}+\mathrm{Na}^{+}$: 503.1980, found: 503.1926 . 


\section{Synthesis of $(1 R, 2 R)-N$-methyl-1,2-diphenyl- $N$-tosylethane-1,2-diamine (7)}<smiles>Cc1ccc(S(=O)(=O)N(C)[C@H](c2ccccc2)[C@H](N)c2ccccc2)cc1</smiles>

Tert-butyl (1R,2R)-2-( $N$-methyl- $N$ - tosylamino)-1,2-diphenylethylcarbamate $(60 \mathrm{mg}, 0.125$ mmol) was added to $\mathrm{CF}_{3} \mathrm{COOH}(0.43 \mathrm{~mL})$ with ice-cooling. After the addition, the reaction mixture was brought to room temperature and stirred for $2 \mathrm{~h}$. TLC analysis indicated completion of the reaction. $\mathrm{CF}_{3} \mathrm{COOH}$ was removed in vacuo. The residue was washed with saturated $\mathrm{NaHCO}_{3}$ and extracted with ethyl acetate. The combined organic phase was concentrated in vacuo. The residue was purified with silica gel chromatography. The pure product was obtained as a white solid in $67 \%$ yield. Mp. $130-132{ }^{\circ} \mathrm{C}$; $[\alpha]^{25} \mathrm{D}+5.0\left(c \mathrm{c} .7, \mathrm{CHCl}_{3}\right)$; IR (KBr) 3382, 1631, 1597, 1383, 1321, 1153, $938 \mathrm{~cm}^{-1}$; ${ }^{1} \mathrm{H}$ NMR ( $\mathrm{CDCl}_{3}$, TMS, $\left.300 \mathrm{MHz}\right) \delta 2.35$ (s, 3H), 2.84 (s, $3 \mathrm{H}), 4.44(\mathrm{~d}, J=10.2 \mathrm{~Hz}, 1 \mathrm{H}), 5.25(\mathrm{~d}, J=10.2 \mathrm{~Hz}, 1 \mathrm{H}), 7.01-7.17(\mathrm{~m}, 12 \mathrm{H}), 7.58(\mathrm{~d}, J=7.2 \mathrm{~Hz}$, $2 \mathrm{H}) ;{ }^{13} \mathrm{C} \mathrm{NMR}\left(\mathrm{CDCl}_{3}\right.$, TMS, $\left.75 \mathrm{MHz}\right) \delta 21.68,29.84,55.79,67.51,127.57,127.97,128.23$, 128.60, 129.24, 129.64, 135.87, 137.10, 142.35, 143.35; HRMS Calcd. for $\mathrm{C}_{22} \mathrm{H}_{24} \mathrm{~N}_{2} \mathrm{O}_{2} \mathrm{~S}+\mathrm{Na}^{+}$: 403.1456, found: 403.1445;

Synthesis of 1-((1R,2R)-2-( $N$-methyl- $N$-tosylamino)-1,2-diphenylethyl)-3-((1R,2R)-2 -(dimethylamino)cyclohexyl)thiourea (1e)

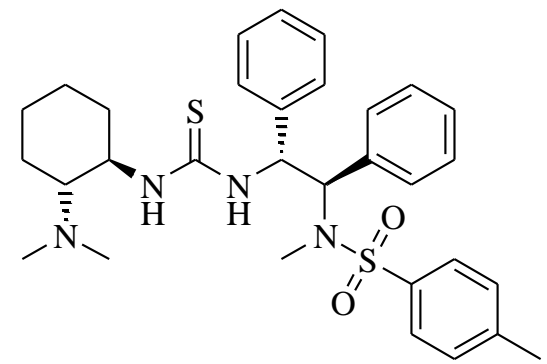

To a solution of $(1 R, 2 R)-N$-methyl-1,2-diphenyl- $N$-tosylethane-1,2-diamine (64 $\mathrm{mg}, 0.168$ $\mathrm{mmol})$ in anhydrous THF $(2 \mathrm{~mL})$ was added $(1 R, 2 R)$-2-isothiocyanato- $N, N-$ dimethylcyclohexanamine $(34 \mathrm{mg}, 0.185 \mathrm{mmol})$ at room temperature. TLC indicated the completion of the reaction after about $4 \mathrm{~h}$. The reaction mixture was concentrated in vacuo and purified with silica gel chromatography. The pure product was obtained as a white solid in $78 \%$ yield. Mp. 80-82 ${ }^{\circ} \mathrm{C} ;[\alpha]^{25} \mathrm{D}-25.0\left(c 0.24, \mathrm{CHCl}_{3}\right)$; IR (KBr) 3373, 3031, 2929, 2857, 1598, 1540, 1326, $1160 \mathrm{~cm}^{-1}$; ${ }^{1} \mathrm{H}$ NMR $\left(\mathrm{CDCl}_{3}\right.$, TMS, $\left.300 \mathrm{MHz}\right) \delta 1.08-1.44(\mathrm{~m}, 4 \mathrm{H}), 1.69-1.91(\mathrm{~m}, 3 \mathrm{H})$, 2.22-2.38 (m, 10H), $2.51(\mathrm{~m}, 1 \mathrm{H}), 2.87(\mathrm{~s}, 3 \mathrm{H}), 3.87(\mathrm{~m}, 1 \mathrm{H}), 5.25(\mathrm{~d}, J=11.7 \mathrm{~Hz}, 1 \mathrm{H}), 6.32-6.39$ $(\mathrm{dd}, J=11.1$ and $8.1 \mathrm{~Hz}, 1 \mathrm{H}), 6.75(\mathrm{br}, 1 \mathrm{H}), 6.92-7.31(\mathrm{~m}, 12 \mathrm{H}), 7.58(\mathrm{~d}, J=8.1,2 \mathrm{H}) ;{ }^{13} \mathrm{C} \mathrm{NMR}$ $\left(\mathrm{CDCl}_{3}, \mathrm{TMS}, 75 \mathrm{MHz}\right) \delta 21.76,24.67,25.28,29.92,30.52,32.94,40.07,54.89$, 57.49, 64.97, 66.70, 127.19, 127.62, 128.27, 128.40, 128.60, 129.47, 129.84, 134.22, 137.48, 139.73, 143.60, 181.76; HRMS Calcd. for $\mathrm{C}_{31} \mathrm{H}_{41} \mathrm{~N}_{4} \mathrm{O}_{2} \mathrm{~S}_{2}$ : 565.2671, found: 565.2582. 


\section{General procedure for asymmetric amination of cyclic $\beta$-keto esters with dialkyl azodicarboxylates with organocatalyst $1 \mathrm{~d}$ :}

The dialkyl azodicarboxylates $3(0.104 \mathrm{mmol})$ in ethyl acetate $(0.2 \mathrm{~mL})$ was added to a vial containing cyclic $\beta$-keto esters $2(0.125 \mathrm{mmol})$ and catalyst $\mathbf{1 d}(7 \mathrm{mg}, 0.0104 \mathrm{mmol})$ in ethyl acetate $(0.3 \mathrm{~mL})$ at $-78^{\circ} \mathrm{C}$. TLC analysis indicated completion of the reaction after about 1-8 $\mathrm{h}$. Then the reaction mixture was concentrated in vacuo to obtain the crude product. The crude product was purified by flash silica gel chromatography to afford the product. 4aa ${ }^{1} \mathrm{H}$ NMR (300 $\left.\mathrm{MHz} \mathrm{CDCl}_{3}\right) \delta 6.88(\mathrm{br}, 1 \mathrm{H}), 4.22-4.28(\mathrm{~m}, 4 \mathrm{H}), 3.86(\mathrm{~s}, 3 \mathrm{H}), 2.75(\mathrm{~m}, 2 \mathrm{H}), 2.40(\mathrm{~m}, 2 \mathrm{H}), 2.11$ $(\mathrm{m}, 1 \mathrm{H}), 1.78(\mathrm{~m}, 1 \mathrm{H}), 1.30-1.37(\mathrm{~m}, 6 \mathrm{H})$; HPLC analysis (Chiralcel AS-H, $i$-PrOH $/ \mathrm{Hexane}=$ 10/90, flow rate: $1 \mathrm{~mL} / \mathrm{min}, \lambda=210 \mathrm{~nm}, \mathrm{t}_{\text {minor }}=24.8 \mathrm{~min}, \mathrm{t}_{\text {major }}=19.3 \mathrm{~min}, 95 \%$ ee.

\section{Acknowledgements}

This work was supported by National Natural Science Foundation of China (20972117), the Fundamental Research Funds for the Central Universities, and SRFDP (J0730426).

\section{References and Notes}

1. (a) Negoro, T.; Murate, M.; Ueda, S.; Fujitani, B.; Ono, Y.; Kuromiya, A.; Suzuki, K.; Matsumoto, J.-I. J. Med. Chem. 1998, 41, 4118. (b) Cativiela, C.; Dîaz-de-Villegas, M. D. Tetrahedron: Asymmetry 1998, 9, 3517. (c) Gibson, S. E.; Guillo, N.; Tozer, M. J. Tetrahedron 1999, 55, 585. (d) Cativiela, C.; Dîaz-de-Villegas, M. D. Tetrahedron: Asymmetry 2000, 11, 645. (e) Bergmeier, S. C. Tetrahedron 2000, 56, 2561.

2. (a) Quaternary Stereocenters: Challenges and Solution for Organic Synthesis Christoffers, J.; Baro A., Eds., Wiley-VCH: Weinheim, 2005. (b) Juji, K. Chem. Rev. 1993, 93, 2037. (c) Corey, E. J.; Guzman-Perez, A. Angew. Chem., Int. Ed. 1998, 37, 388. (d) Christoffers, J.; Mann, A. Angew. Chem., Int. Ed. 2001, 40, 4591. (e) Denissova, I.; Barriault, L. Tetrahedron 2003, 59, 10105. (f) Christoffers, J.; Baro, A. Adv. Synth. Catal. 2005, 347, 1473. (g) Riant, O.; Hannedouche, J. Org. Biomol. Chem. 2007, 5, 873. (h) Chavarot, M.; Byrne, J. J.; Chavant, P. Y.; Vallee, Y. Tetrahedron: Asymmetry 2001, 12, 1147.

3. (a) Marigo, M.; Juhl, K.; Jørgensen, K. A. Angew. Chem. Int. Ed. 2003, 42, 1367. (b) Foltz, C.; Stecker, B.; Marconi, G.; Bellemin-Laponnaz, S.; Wadepohl H.; Gade, L. H. Chem. Commun. 2005, 5115. (c) Ma, S.; Jiao, N.; Zheng, Z.; Ma, Z.; Lu, Z.; Ye, L.; Deng, Y.; Chen, G. Org. Lett. 2004, 6, 2193. (d) Huber, D. P.; Stanek, K.; Togni, A. Tetrahedron: Asymmetry 2006, 17, 658.

4. Mashiko, T.; Hara, K.; Tanaka, D.; Fujiwara, Y.; Kumagai, N.; Shibasaki, M. J. Am. Chem. Soc. 2007, 129, 11342. 
5. For selected reviews of organocatalysis, see: (a) Dalko, P. I.; Moisan, L. Angew. Chem. Int. Ed. 2001, 40, 3726. (b) Dalko, P. I.; Moisan, L. Angew. Chem. Int. Ed. 2004, 43, 5138. (c) Acc. Chem. Res. 2004, 37, 487. Special issue on organocatalysis. (d) Berkessel, A.; Gröger, H.; Asymmetric Organocatalysis: From Biomimetic Concepts to Applications in Asymmetric Synthesis, Wiley-VCH: Weinheim 2004. (e) Seayad J.; List, B. Org. Biomol. Chem. 2005, 3, 719. (f) Dondoni, A.; Massi, A. Angew. Chem. Int. Ed. 2008, 47, 4638.

6. Pihko, P. M.; Pohjakallio, A. Synlett 2004, 12, 2115.

7. (a) Saaby, S.; Bella, M.; Jørgensen, K. A. J. Am. Chem. Soc. 2004, 126, 8120. (b) Liu, X.; Li, H.; Deng, L. Org. Lett. 2005, 7, 167. For asymmetric amination of aldehydes, ketones, $\alpha-$ keto esters and alkylidene cyanoacetates, see: (c) List, B. J. Am. Chem. Soc. 2002, 124, 5656. (d) Bøgevig, A.; Juhl, K.; Kumaragurubaran, N.; Zhuang, W.; Jørgensen, K. A. Angew. Chem. Int. Ed. 2002, 41, 1790. (e) Vogt, H.; Vanderheiden, S.; Bräse, S. Chem. Commun. 2003, 2448. (f) Iwamura, H.; Mathew, S. P.; Blackmond, D. G. J. Am. Chem. Soc. 2004, 126, 11770. (g) Kumaragurubaran, N.; Juhl, K.; Zhuang, W.; Bøgevig, A.; Jørgensen, K. A. J. Am. Chem. Soc. 2002, 124, 6254. (h) Juhl, K.; Jørgensen, K. A. J. Am. Chem. Soc. 2002, 124, 2420. (i) Poulsen, T. B.; Alemparte, C.; Jørgensen, K. A. J. Am. Chem. Soc. 2005, 127, 11614. (j) Guo, H.-M.; Cheng, L.; Cun, L.-F.; Gong, L.-Z.; Mi, A.-Q.; Jiang, Y.Z. Chem. Commun. 2006, 429. (k) Liu, T.-Y.; Cui, H.-L.; Zhang, Y.; Jiang, K.; Du, W.; He, Z.-Q.; Chen, Y.-C. Org. Lett. 2007 9, 3671.

8. Xu, X.; Yabuta, T.; Yuan, P.; Takemoto, Y. Synlett 2006, 137.

9. (a) Terada, M.; Nakano, M.; Ube, H. J. Am. Chem. Soc. 2006, 128, 16044. (b) He, R.; Wang, X.; Hashimoto, T.; Maruoka, K. Angew. Chem. Int. Ed. 2008, 47, 9466.

10. (a) Wang, C.-J.; Zhang, Z.-H.; Dong, X.-Q.; Wu, X.-J. Chem. Comm. 2008, 1431. (b) Zhang, Z.-H.; Dong, X.-Q.;Chen, D; Wang, C.-J. Chem.-Eur. J. 2008, 14, 8780. (c) Wang, C.-J.; Dong, X.-Q.; Zhang, Z.-H.; Xue, Z.-Y.; Teng, H.-L. J. Am. Chem. Soc. 2008, 130, 8606. (d) Dong, X.-Q.; Teng, H.-L.; Wang, C.-J. Org. Lett. 2009, 11, 1265.

11. This was probably caused by the unstability of the amine-thiourea catalyst under the reaction condition, which was also observed by Takemoto et al, see Ref. 8. 